\title{
Relativistic Brownian motion: From a microscopic binary collision model to the Langevin equation
}

\author{
Jörn Dunkel ${ }^{1, \text { 困 }}$ and Peter Hänggi ${ }^{1}$ \\ ${ }^{1}$ Institut für Physik, Universität Augsburg, Theoretische Physik I, \\ Universitätstraße 1, D-86135 Augsburg, Germany
}

(Dated: August 3, 2006)

\begin{abstract}
The Langevin equation (LE) for the one-dimensional relativistic Brownian motion is derived from a microscopic collision model. The model assumes that a heavy point-like Brownian particle interacts with the lighter heat bath particles via elastic hard-core collisions. First, the commonly known, non-relativistic LE is deduced from this model, by taking into account the non-relativistic conservation laws for momentum and kinetic energy. Subsequently, this procedure is generalized to the relativistic case. There, it is found that the relativistic stochastic force is still $\delta$-correlated (white noise) but does no longer correspond to a Gaussian white noise process. Explicit results for the friction and momentum-space diffusion coefficients are presented and elucidated.
\end{abstract}

PACS numbers: $\quad 05.40 .-\mathrm{a}, 02.50 . E y$, 05.40.Jc, 47.75.+f

\section{INTRODUCTION}

The theories of the non-relativistic Brownian motion and special relativity were introduced more than 100 years ago [1, 2, 3, 4, 5, 6, 7]. Since then, they have become cornerstones for our understanding of a wide range of physical processes [8, 9, 10, 11, 12]. This fact notwithstanding, the unification of both concepts poses a theoretical challenge still nowadays (classical references are 13, 14, 15, 16, 17, 18]; recent contributions include 19, 20, 21, 22, 23, 24, 25, 26, 27, 28, 29, 30, 31]; potential applications in high-energy physics and astrophysics are considered in [32, 33, 34, 35, 36, 37]). The relatively slow progress in this field can be attributed to the severe difficulties that arise when one tries to describe $N$-body systems in a relativistically consistent manner [38, 39]. Due to this reason, the derivation of relativistic Langevin equations (LEs) from an underlying microscopic model has remained an unsolved issue until now 63]. However, in the present paper we aim to provide a solution to this problem.

More precisely, by considering quasi-elastic, binary collisions between the Brownian and heat bath particles [40, 41] we are able to treat the heat bath in a fully relativistic manner without having to account for the exact details of the relativistic $N$-body interactions. As shown in Sec. III for a non-relativistic framework this approach yields the well-known non-relativistic LE with Gaussian white noise as well as the correct fluctuation-dissipation theorem (the Einstein-Sutherland relation 7]). In Sec. IIII the method is transferred to the relativistic case, leading to the main result of this paper, the relativistic LE (32). Remarkably, the relativistic stochastic force is also $\delta$-correlated ('white') but no longer of Gaussian (or Wiener [42]) type. Compared with the

*Electronic address: joern.dunkel@physik.uni-augsburg.de non-relativistic Brownian motion, this is the most important difference. Furthermore, we obtain explicit representations of friction and (momentum)-diffusion coefficients in terms of expectation values with respect to the heat bath distribution (see also Appendix A).

\section{NON-RELATIVISTIC BROWNIAN MOTIONS}

The objective of this section is to recover the wellknown non-relativistic LEs from a simple microscopic collision model for Brownian motions. As is well known 43, 44, 45, 46, 47], non-relativistic LEs can also be derived by considering a bath of harmonic oscillators possessing a canonical phase space distribution. Unfortunately, however, it is problematic to transfer this approach to the relativistic case, since the instantaneous linear (and nonlinear) interactions between Brownian and heat particles violate the basic principles of special relativity. To circumvent this problem, we will pursue a different method here, using only the (non-)relativistic microscopic conservation laws for energy and momentum, respectively, known to hold for elastic point-like, binary collisions. Conceptually, our approach is related to that of Pechukas [40] and Pechukas-Tsonchev [4], who considered a similar model in the context of non-relativistic quantum Brownian motion [48] (similar approaches are also known from unimolecular rate theory, see e.g. Sec. V in [4]]).

\section{A. Microscopic model}

For the sake of simplicity only, we will restrict ourselves throughout to the one-dimensional (1d) case. Generalizations to higher space dimensions are in principle straightforward, but certain calculations will become much more cumbersome (cf. corresponding comments in Appendix A). To start out, consider the following 
situation in the inertial laboratory frame $\Sigma_{0}$ : A large one-dimensional box volume $\mathcal{V} \equiv[-L / 2, L / 2]$ contains an ideal non-relativistic gas, consisting of $N$ small pointlike particles with identical masses $m$. The gas particles - referred to as 'heat bath' hereafter - surround a Brownian particle of mass $M \gg m$. Due to frequent elastic collisions with heat bath particles, the Brownian particle performs stochastic motions.

\section{Heat bath}

The coordinates and momenta of the heat bath particles are denoted by $x_{r} \in[-L / 2, L / 2]$ and $p_{r} \in(-\infty ; \infty)$, respectively, where $r=1, \ldots, N$. As usual, we make the following simplifying assumption concerning the heat bath: The probability density function (PDF) of the heat bath particles is a spatially homogeneous Maxwell distribution, i.e., at each time $t>0$, the $\mathrm{PDF}$ reads

$$
f_{\mathrm{b}}^{N}\left(x_{1}, \ldots, p_{N}\right)=\left(\frac{\lambda}{L}\right)^{N} \prod_{r=1}^{N} \exp \left(-\frac{p_{r}^{2}}{2 m k T}\right),
$$

where $k$ is the Boltzmann constant, $T$ the temperature, and $\lambda=(2 \pi m k T)^{-1 / 2}$. Thus, it is implicitly assumed that:

- the heat bath particles are independently and identically distributed;

- the collisions with the Brownian particle do not significantly alter the bath distribution.

These assumptions are justified, if the collisions between the gas particles rapidly reestablish a spatially homogeneous distribution.

\section{Kinematics of single collision events}

The momentum and energy balance per (elastic) collision reads

$$
E+\epsilon=\hat{E}+\hat{\epsilon}, \quad P+p=\hat{P}+\hat{p} .
$$

Here and below, capital letters refer to the Brownian particle and small letters to particles forming the heat bath; quantities without (with) hat-symbols refer to the state before (after) the collision. In the non-relativistic case, we have, e.g., before the collision

$$
P=M V, \quad p=m v, \quad E=\frac{P^{2}}{2 M}, \quad \epsilon=\frac{p^{2}}{2 m},
$$

where $v$ and $V$ denote the velocities. Taking into account both conservation of momentum and (kinetic) energy, one finds that the change $\Delta P \equiv \hat{P}-P$ of the Brownian particle's momentum per single collision is given by

$$
\Delta P=\frac{-2 m}{M+m} P+\frac{2 M}{M+m} p .
$$

\section{B. Derivation of the Langevin equation}

The total momentum change $\delta P$ of the Brownian particle within the time interval $\tau$ can be written as

$$
\delta P(t) \equiv P(t+\tau)-P(t)=\sum_{i=1}^{N} \Delta P_{r} I_{r}(t, \tau),
$$

where $I_{r}(t, \tau) \in\{0,1\}$ is the indicator function for a collision with the heat bath particle $r$ during the interval $[t, t+\tau]$; i.e. $I_{r}(t, \tau)=1$ if a collision has occurred, and, otherwise, $I_{r}(t, \tau)=0$. In the $1 d$ case, the collision indicator can be written explicitly as

$$
\begin{aligned}
I_{r}(t, \tau)= & \Theta\left(X-x_{r}\right) \Theta\left(x_{r}^{\prime}-X^{\prime}\right) \Theta\left(v_{r}-V\right)+ \\
& \Theta\left(x_{r}-X\right) \Theta\left(X^{\prime}-x_{r}^{\prime}\right) \Theta\left(V-v_{r}\right),
\end{aligned}
$$

where $X=X(t), x_{r}=x_{r}(t)$, and

$$
X^{\prime}=X+V \tau, \quad x_{r}^{\prime}=x_{r}+v_{r} \tau
$$

are the projected particle positions at time $t+\tau$. The Heaviside-function is defined by

$$
\Theta(x)= \begin{cases}0, & x<0 \\ 1 / 2, & x=0 \\ 1, & x>0\end{cases}
$$

The expectation $\left\langle I_{r}(t, \tau)\right\rangle_{\mathrm{b}}$ gives the probability that the bath particle $r$ collides with the Brownian particle between $t$ and $t+\tau$. As shown in Appendix $\mathrm{A}$ in the limit $\tau \rightarrow 0$, one finds

$$
\left\langle I_{r}(t, \tau)\right\rangle_{\mathrm{b}}=\tilde{C}(V) \frac{\tau}{L}=C(P) \frac{\tau}{L},
$$

with function $C(P)=\tilde{C}(V(P))$ given by the integral formula

$$
\begin{aligned}
\tilde{C}(V) \equiv & \frac{1}{2} \int_{V}^{\infty} \mathrm{d} v_{r}\left(v_{r}-V\right) \tilde{f}_{\mathrm{b}}^{1}\left(v_{r}\right)+ \\
& \frac{1}{2} \int_{-\infty}^{V} \mathrm{~d} v_{r}\left(V-v_{r}\right) \tilde{f}_{\mathrm{b}}^{1}\left(v_{r}\right) .
\end{aligned}
$$

Here, $\tilde{f}_{\mathrm{b}}^{1}\left(v_{r}\right)$ is the one-particle velocity PDF of a heat bath particle. We anticipate that Eqs. (6) and (7) remain valid in the relativistic case as well.

However, in order to recover from Eqs. (5) - (7) the wellknown non-relativistic LE, we still have to make a number of simplifying assumptions:

(i) The time interval $\tau$ is sufficiently small, so that $|\delta P / P| \ll 1$. In particular, $\tau$ is supposed to be so small that there occurs at most only one collision between the Brownian particle and a specific heat bath particle $r$. One the other hand, the time interval $\tau$ should still be large enough, so that the total number of collisions within $\tau$ is larger than 1 . These requirements can be fulfilled simultaneously only if $m / M \ll 1$. 
(ii) Collisions occurring within $[t, t+\tau]$ can be viewed as independent events.

(iii) Finally, we will (have to) assume that

$$
\begin{aligned}
\left.\left\langle p_{r} I_{r}(t, \tau)\right]^{j}\right\rangle_{\mathrm{b}} & =\left\langle p_{r}^{j} I_{r}(t, \tau)\right\rangle_{\mathrm{b}} \\
& \simeq\left\langle p_{r}^{j}\right\rangle_{\mathrm{b}}\left\langle I_{r}(t, \tau)\right\rangle_{\mathrm{b}} \\
& =\left\langle p_{r}^{j}\right\rangle_{\mathrm{b}} C(P) \frac{\tau}{L}
\end{aligned}
$$

for $j=1,2, \ldots$. Given the explicit representation of the indicator function (6a), it is in principle straightforward to check the quality of the approximation (8), if a bath distribution has been specified.

As we shall see immediately, the assumptions (i)-(iii) are necessary and sufficient for deriving the well-known non-relativistic LE from Eqs. (5)-(7). Upon inserting Eq. (4) into (5) and dividing by $\tau$ we find

$$
\begin{aligned}
\frac{\delta P(t)}{\tau} \simeq- & {\left[\frac{1}{\tau} \sum_{r=1}^{N} \frac{2 m}{m+M} I_{r}(t, \tau)\right] P+} \\
& \frac{1}{\tau} \sum_{r=1}^{N} \frac{2 M}{M+m} p_{r} I_{r}(t, \tau) .
\end{aligned}
$$

The first term on the rhs. in Eq. (9) can be identified as the 'friction' term, whereas the second term represents 'noise'. On the rhs. of Eq. (9), it was assumed that for each collision occurring within $[t, t+\tau]$, the initial momentum of the Brownian particle is approximately equal to some suitably chosen value $P\left(t^{\prime}\right)$ with $t^{\prime} \in[t, t+\tau]$, cf. the assumption (i) above and the discussion at the end of this section.

The next step en route to the conventional LE consists in replacing the square bracket expression in Eq. (9) by the averaged friction coefficient

$$
\nu_{0} \equiv \frac{1}{\tau} \sum_{r=1}^{N} \frac{2 m}{m+M}\left\langle I_{r}(t, \tau)\right\rangle_{\mathrm{b}} .
$$

Since it was assumed that the heat bath particles are independently and identically distributed, we can rewrite this as

$$
\nu_{0}=\frac{N}{\tau} \frac{2 m}{m+M}\left\langle I_{r}(t, \tau)\right\rangle_{\mathrm{b}}
$$

for some $r \in\{1, \ldots, N\}$. The coefficient $\nu_{0}$ can be interpreted as an average collision rate weighted by some mass ratio. Inserting Eq. (7a) into Eq. (10b) yields

$$
\nu_{0}=n_{\mathrm{b}} \frac{2 m}{m+M} C(P),
$$

where $n_{\mathrm{b}}=N / L$ is the density of the bath particles. In the case of the Maxwell distribution, we can evaluate the integral (7b), and find

$$
\begin{gathered}
C(P)=\left(\frac{k T}{2 \pi m}\right)^{1 / 2} \exp \left[-\frac{m}{2 k T}\left(\frac{P}{M}\right)^{2}\right]+ \\
\frac{P}{2 M} \operatorname{erf}\left[\left(\frac{m}{2 k T}\right)^{1 / 2} \frac{P}{M}\right] .
\end{gathered}
$$

In particular, setting (see Appendix A)

$$
C(P) \approx C(0)=\left(\frac{k T}{2 \pi m}\right)^{1 / 2}
$$

corresponds to the commonly used Stokes approximation. It then remains to analyze the 'noise force'

$$
\xi(t) \equiv \frac{1}{\tau} \sum_{r=1}^{N} \frac{2 M}{M+m} p_{r} I_{r}(t, \tau),
$$

corresponding to the last term in Eq. (9). Averaging over the bath distribution $f_{\mathrm{b}}^{N}$ and using Eqs. (8), we find for the mean value

$$
\langle\xi(t)\rangle_{\mathrm{b}}=0
$$

Furthermore, assuming mutual independence of the collisions, the correlation function is obtained as

$$
\begin{aligned}
\langle\xi(t) \xi(s)\rangle_{\mathrm{b}} & =\frac{\delta_{t s}}{\tau^{2}}\left(\frac{2 M}{M+m}\right)^{2} \sum_{r=1}^{N}\left\langle p_{r}^{2} I_{r}^{2}(t, \tau)\right\rangle_{\mathrm{b}} \\
& \stackrel{\text { 8 }}{\simeq} \frac{\delta_{t s}}{\tau^{2}}\left(\frac{2 M}{M+m}\right)^{2} \sum_{r=1}^{N} m k T\left\langle I_{r}(t, \tau)\right\rangle_{\mathrm{b}} \\
& \stackrel{\text { 10a }}{=} \frac{\delta_{t s}}{\tau}\left(\frac{2 M^{2}}{M+m}\right) \nu_{0} k T,
\end{aligned}
$$

with $\delta_{t s} \in\{0,1\}$ denoting the Kronecker-symbol. To obtain the second line, we have used that $I_{r}^{2}(t, \tau)=I_{r}(t, \tau)$, and the simplifying assumption (8) that $I_{r}(t, \tau)$ and $p_{r}$ are (approximately) independent random variables with respect to the bath distribution.

Similar to Eq. (14b), also the higher correlation functions are determined by the corresponding moments of the Gaussian marginal bath distribution (11). Thus, under the above assumptions (i)-(iii), the non-relativistic stochastic force $\xi(t)$ corresponds to Gaussian white noise (or a Wiener process [42], respectively).

Finally, by substituting $\nu_{0}$ from Eq (11) for the square bracket expression in Eq. (9) and formally letting $\tau \rightarrow 0$, we recover from Eq. (9) the well-known non-relativistic LE 47, 50, 51

$$
\dot{P}=-\nu_{0} P+\xi(t),
$$

where $\xi$ is a Gaussian white noise force, characterized by

$$
\begin{aligned}
\langle\xi(t)\rangle & =0, \\
\langle\xi(t) \xi(s)\rangle & =2 D_{0} \delta(t-s),
\end{aligned}
$$

with (momentum-space) diffusion coefficient

$$
D_{0}=\frac{M^{2}}{M+m} \nu_{0} k T
$$

Here, we used that $\delta_{s t} / \tau \rightarrow \delta(t-s)$ for $\tau \rightarrow 0$, where $\delta(t-s)$ is the Dirac-function. 
In the limit $m / M \rightarrow 0$, Eq. (15d) reduces to the standard fluctuation-dissipation theorem $D_{0}=M \nu_{0} k T$ [7, 52]. However, $\nu_{0}$ and $D_{0}$ are constants only if one adopts the Stokes approximation (12), cf. Appendix A If one goes beyond the Stokes approximation, then the noise in Eqs. (15) becomes multiplicative with respect to $P$, and, therefore, Eqs. (15) must be complemented by a discretization rule in this case $50,53,54,55,56,57$, 58, 59, 60]. As discussed in 50, 58, 59, 60], it is only for the post-point discretization rule, corresponding to $\nu_{0}(P)=\nu_{0}(P(t+\tau))$ and $D_{0}(P)=D_{0}(P(t+\tau))$ on the rhs. of Eq. 15a), one recovers the Maxwellian PDF

$$
\Phi_{\infty}(P)=\left(\frac{1}{2 \pi M k T}\right)^{1 / 2} \exp \left(-\frac{P^{2}}{2 M k T}\right)
$$

as the stationary momentum distribution of the Brownian particle in the limit $t \rightarrow \infty$ (assuming that $m / M \rightarrow$ $0)$.

\section{RELATIVISTIC BROWNIAN MOTIONS}

We shall now apply an analogous reasoning to obtain a relativistic LE. For this purpose we consider an inertial (laboratory) frame $\Sigma_{0}$ with time coordinate $t$, as e.g. measured by an atomic clock resting in $\Sigma_{0}$.

\section{A. Microscopic model}

The basic constituents of the microscopic model are the same as those outlined in Sec. IIA but in addition we now have to consider a relativistic heat bath distribution and must consistently take into account the relativistic collision kinematics.

\section{Relativistic heat bath}

In the relativistic case, we postulate analogous to Eq. (11) that, with respect to $\Sigma_{0}$, the heat bath distribution is stationary, spatially homogeneous, and independent, so that the PDF can be written in the product form

$$
f_{\mathrm{b}}^{N}\left(x_{1}, \ldots, p_{N}\right)=L^{-N} \prod_{r=1}^{N} f_{\mathrm{b}}^{1}\left(p_{r}\right) .
$$

As marginal one-particle momentum PDFs, we will now consider the $\eta$-generalized Jüttner-Maxwell distributions [31, 61], reading:

$$
f_{\mathrm{b}}^{1}(p)=\frac{\mathcal{N}_{\eta}}{\epsilon(p)^{\eta}} \exp \left[-\frac{\epsilon(p)}{k T}\right], \quad \eta \geq 0,
$$

where $p \in(-\infty,+\infty)$, and $\epsilon(p)$ denotes the relativistic kinetic energy of a heat bath particle. The normalization constant $\mathcal{N}_{\eta}$ is determined by

$$
\mathcal{N}_{\eta}^{-1}=\int_{-\infty}^{\infty} \mathrm{d} p f_{\mathrm{b}}^{1}(p)
$$

For $\eta=0$, Eq. 17b reduces to the standard JüttnerMaxwell distribution 61]. However, as discussed recently [31, 37], the PDF with $\eta=1$ appears to be conserved in relativistic elastic binary collisions. In general, however, the arguments and results presented below remain valid for arbitrary one-particle momentum PDFs $f_{\mathrm{b}}^{1}(p)$, i.e., also for momentum distributions other than the $\eta$-generalized Jüttner PDFs (17b).

\section{Relativistic collision kinematics}

Using natural units such that $c=1$, relativistic kinetic energy, momentum and velocity are related by

$$
\begin{aligned}
p & =m v \gamma(v), & \epsilon(p) & =\left(m^{2}+p^{2}\right)^{1 / 2}, \\
P & =M V \gamma(V), & E(P) & =\left(M^{2}+P^{2}\right)^{1 / 2},
\end{aligned}
$$

where $\gamma(v) \equiv\left(1-v^{2}\right)^{-1 / 2}$. As before, capital letters refer to the Brownian particle. Inserting Eq. (18) into the conservation laws (2), and solving for $\hat{P}$, one finds 31]

$$
\hat{P}=\frac{2 u E-\left(1+u^{2}\right) P}{1-u^{2}}
$$

where

$$
u(p, P)=\frac{P+p}{E+\epsilon}
$$

is the center-of-mass velocity. Hence, the momentum change $\Delta P=\hat{P}-P$ of the Brownian particle during a single collision is given by

$$
\Delta P=-\frac{2}{1-u^{2}} \frac{\epsilon}{E+\epsilon} P+\frac{2}{1-u^{2}} \frac{E}{E+\epsilon} p .
$$

In the non-relativistic limit case, where $u^{2} \ll 1, E \simeq M$ and $\epsilon \simeq m$, this reduces to Eq. (4).

\section{B. Derivation of the Langevin equation}

Inserting Eq. (21) into Eq. (5), one obtains the relativistic analogon of Eq. (9) as

$$
\begin{aligned}
\frac{\delta P(t)}{\tau} \simeq-[ & \left.\frac{1}{\tau} \sum_{r=1}^{N} \frac{2}{1-u_{r}^{2}} \frac{\epsilon_{r}}{E+\epsilon_{r}} I_{r}(t, \tau)\right] P+ \\
& \frac{1}{\tau} \sum_{r=1}^{N} \frac{2}{1-u_{r}^{2}} \frac{E}{E+\epsilon_{r}} p_{r} I_{r}(t, \tau),
\end{aligned}
$$

where $u_{r} \equiv u\left(p_{r}, P\right)$ and $\epsilon_{r} \equiv \epsilon\left(p_{r}\right)$. Formally, the collision indicator $I_{r}(t, \tau)$ is still determined by Eqs. (6) and 
(7), but differences arise due to the fact that we have to use $V=P /\left(M^{2}+P^{2}\right)^{1 / 2}$ and a relativistic bath distribution now.

Analogous to the non-relativistic case, we can identify the first term on the rhs. of Eq. (22) as friction, and introduce an averaged friction coefficient by

$$
\begin{aligned}
\nu(P) & \equiv \frac{1}{\tau} \sum_{r=1}^{N}\left\langle\frac{2}{1-u_{r}^{2}} \frac{\epsilon_{r}}{E+\epsilon_{r}} I_{r}(t, \tau)\right\rangle_{\mathrm{b}} \\
& =\frac{N}{\tau}\left\langle\frac{2}{1-u_{r}^{2}} \frac{\epsilon_{r}}{E+\epsilon_{r}} I_{r}(t, \tau)\right\rangle_{\mathrm{b}},
\end{aligned}
$$

for some $r \in\{1, \ldots, N\}$. Next, applying a product approximation similar to (8), we obtain

$$
\begin{aligned}
& \nu(P) \simeq \frac{N}{\tau}\left\langle\frac{2}{1-u_{r}^{2}} \frac{\epsilon_{r}}{E+\epsilon_{r}}\right\rangle_{\mathrm{b}}\left\langle I_{r}(t, \tau)\right\rangle_{\mathrm{b}} \\
& \stackrel{(7}{=} n_{\mathrm{b}} C(P)\left\langle\frac{2}{1-u_{r}^{2}} \frac{\epsilon_{r}}{E+\epsilon_{r}}\right\rangle_{\mathrm{b}} \text {, }
\end{aligned}
$$

where $n_{\mathrm{b}}=N / L$ is the density of the heat bath particles, and $C(P)$ is determined by Eq. (7b). Figure 1 shows the $P$-dependence of $\nu(P) /\left[n_{\mathrm{b}} C(P)\right]$ for the bath distributions from Eq. (17b). This momentum dependence is induced by the appearance of $u_{r}=u\left(p_{r}, P\right)$ and $E=E(P)$ in the expectation value on the rhs. of Eq. (24). Furthermore, the shape of the one-particle collision coefficient $C(P)=\left\langle I_{r}(t, \tau)\right\rangle_{\mathrm{b}} L / \tau$ is depicted in Fig. 2 As one would intuitively expect, the friction coefficient grows with the temperature $T$ of the heat bath (at constant $P$ ) as well as with the absolute momentum of the Brownian particle (at constant $T$ ).

At this point, it might be worthwhile to emphasize once again that product approximations of the form

$$
\left\langle G\left(x_{r}, p_{r}\right) I_{r}(t, \tau)\right\rangle_{\mathrm{b}} \simeq\left\langle G\left(x_{r}, p_{r}\right)\right\rangle_{\mathrm{b}}\left\langle I_{r}(t, \tau)\right\rangle_{\mathrm{b}},
$$

as employed in Eq. (8) and also in the first line of Eq. (24), can in principle be omitted by using the explicit representation (6) of the collision indicator and the Eqs. (A6) of the Appendix; if one opts to avoid such approximations then the accuracy of the Langevin model increases (note that this statement applies to the nonrelativistic case, too). However, in the following we shall continue to use Eq. (25) in order to obtain a relativistic LE that is on an equal footing with the non-relativistic LE (15).

For this purpose, we interpret the second term on the rhs. of Eq. (22) as 'noise', defining

$$
\chi(t) \equiv \frac{1}{\tau} \sum_{r=1}^{N} \frac{2}{1-u_{r}^{2}} \frac{E}{E+\epsilon_{r}} p_{r} I_{r}(t, \tau) .
$$

Averaging over the bath distribution $f_{\mathrm{b}}^{N}$, one finds for
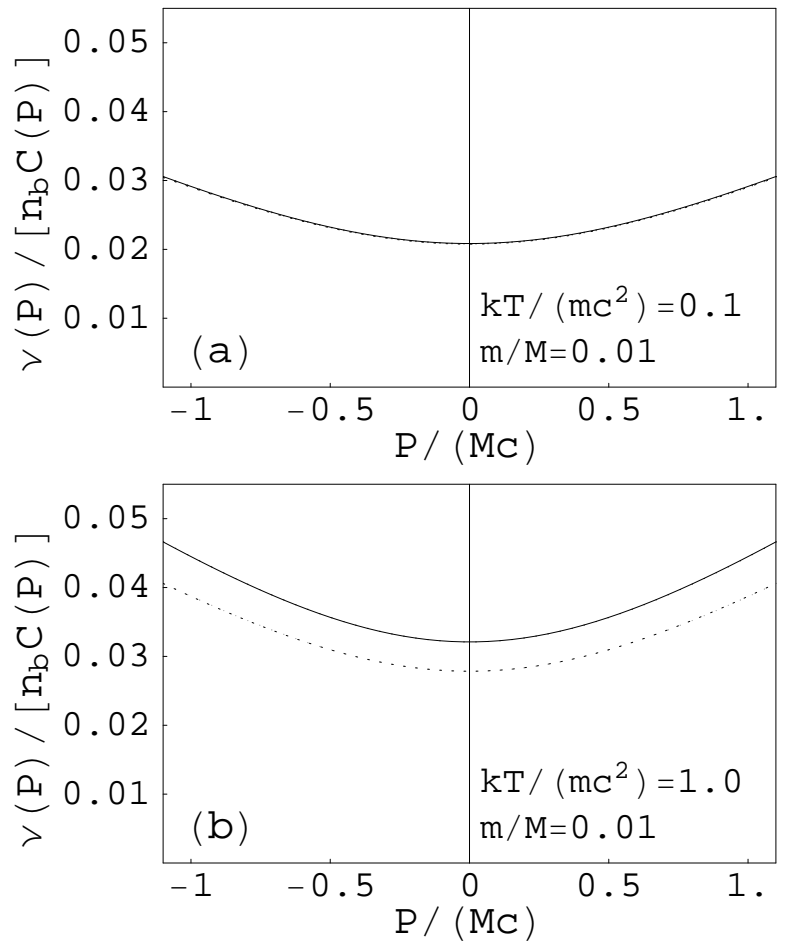

FIG. 1: The momentum-dependent, relativistic friction coefficient $\nu(P)$, divided by the total mean collision rate, $\nu(P) /\left[n_{\mathrm{b}} C(P)\right]$, as calculated numerically for two different heat bath distributions $f_{\mathrm{b}}^{1}(p)$ and two different bath temperatures is depicted versus the scaled momentum $P$. The solid lines refer to the standard Jüttner distribution with $\eta=0$, and the dotted lines to $\eta=1$ in Eq. (17b). (a) Weakly relativistic heat bath. In the limit $k T \ll m c^{2}$ the bath distributions (17b) approach a Maxwellian, and therefore the results for different $\eta$ practically coincide. In particular, for $P=0$ the non-relativistic result is recovered. (b) Strongly relativistic heat bath. The friction coefficient increases with the temperature of the heat bath.

the mean value

$$
\begin{aligned}
\mu(P) & \equiv\langle\chi(t)\rangle_{\mathrm{b}} \\
& =\frac{N}{\tau}\left\langle\frac{2}{1-u_{r}^{2}} \frac{E}{E+\epsilon_{r}} p_{r} I_{r}(t, \tau)\right\rangle_{\mathrm{b}} \\
& \stackrel{\text { 25 }}{\simeq} \frac{N}{\tau}\left\langle\frac{2}{1-u_{r}^{2}} \frac{E}{E+\epsilon_{r}} p_{r}\right\rangle_{\mathrm{b}}\left\langle I_{r}(t, \tau)\right\rangle_{\mathrm{b}} \\
& \simeq n_{\mathrm{b}} C(P)\left\langle\frac{2}{1-u_{r}^{2}} \frac{E}{E+\epsilon_{r}} p_{r}\right\rangle_{\mathrm{b}} .
\end{aligned}
$$

In contrast to the non-relativistic case, the mean value $\mu$ of the relativistic Langevin force $\chi(t)$ depends on the momentum $P$ of the Brownian particle. This can be attributed to the appearance of $u_{r}^{2}=\left(P+p_{r}\right)^{2} /\left(E+\epsilon_{r}\right)^{2}$ in Eq. (27). As shown in Fig. 3] the quantity $\mu(P) /\left[n_{\mathrm{b}} C(P)\right]$ is positive for $P>0$ and negative for $P<0$. Thus, on average, the relativistic stochastic force tends to accelerate particles in the direction of their motion, but this effect is compensated by the increase of the 

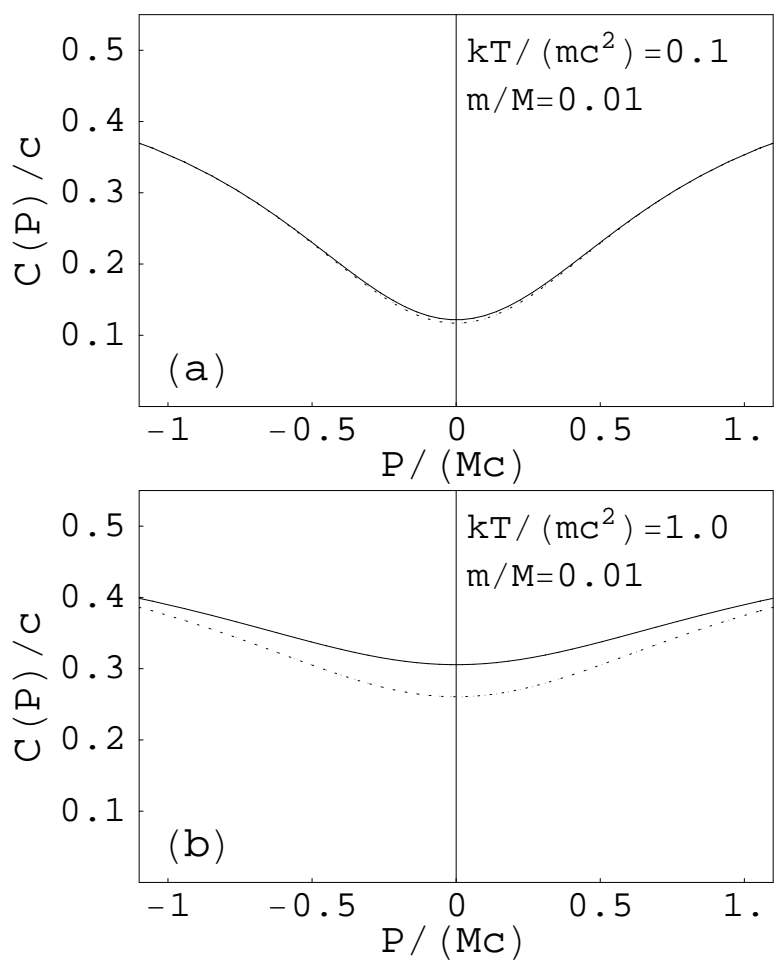

FIG. 2: Relativistic one-particle collision coefficient $C(P)=$ $\left\langle I_{r}(t, \tau)\right\rangle_{\mathrm{b}} L / \tau$, numerically calculated for the same parameters as in Fig. 11 The solid lines refer to a standard Jüttner distribution with $\eta=0$, and the dotted lines to $\eta=1$ in Eq. 17b. (a) Weakly relativistic heat bath. At small temperatures, the zero-value $C(0)$ is approximately equal to the non-relativistic Stokes value $\sqrt{k T /(2 \pi m)}$. (b) Strongly relativistic heat bath. For $|P| \rightarrow \infty$ the coefficient $C(P)$ converges to $1 / 2$.

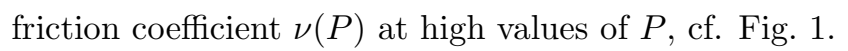

Let us next take a closer look at the correlation function

$$
\sigma_{t s} \equiv\langle\chi(t) \chi(s)\rangle_{\mathrm{b}}-\langle\chi(t)\rangle_{\mathrm{b}}\langle\chi(s)\rangle_{\mathrm{b}}
$$

In the non-relativistic case, the second (product) term vanishes, because the non-relativistic stochastic force possesses a vanishing mean value, $\langle\xi(t)\rangle_{\mathrm{b}}=0$. According to Eq. (27), this is no longer the case for the relativistic noise force $\chi(t)$. In order to explicitly calculate $\sigma_{t s}$, it is convenient to introduce the abbreviation

$$
\kappa_{r}=\frac{2}{1-u_{r}^{2}} \frac{E}{E+\epsilon_{r}} p_{r}
$$

Assuming, as before, that collisions can be viewed as independent events, the correlation function (28) vanishes
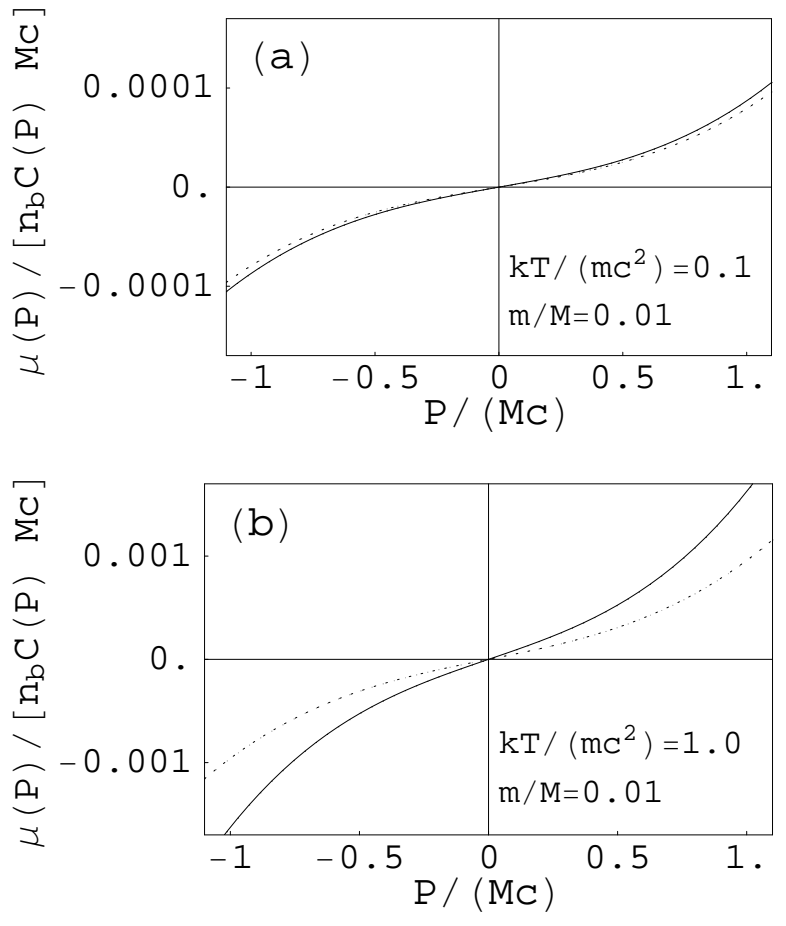

FIG. 3: Mean value of the relativistic stochastic force, $\mu(P) \equiv\langle\chi(t)\rangle_{\mathrm{b}}$, calculated numerically for two different heat bath distributions $f_{\mathrm{b}}^{1}(p)$ and two different bath temperatures. Solid lines refer to a standard Jüttner distribution with $\eta=0$, and dotted lines to $\eta=1$ in Eq. 17b.

at non-equal times $t \neq s$, and we thus find

$$
\begin{aligned}
& \sigma_{t s} \simeq \delta_{t s}\left\{\left\langle\left[\frac{1}{\tau} \sum_{r=1}^{N} \kappa_{r} I_{r}(t, \tau)\right]^{2}\right\rangle_{\mathrm{b}}-\mu^{2}(P)\right\} \\
& \stackrel{25.5}{\simeq} \frac{\delta_{t s}}{\tau^{2}}\left\{\sum_{r=1}^{N}\left\langle\kappa_{r}^{2}\right\rangle_{\mathrm{b}}\left\langle I_{r}(t, \tau)\right\rangle_{\mathrm{b}}-\tau^{2} \mu^{2}(P)+\right. \\
& \left.\sum_{r=1}^{N} \sum_{j \neq r}^{N}\left\langle\kappa_{r}\right\rangle_{\mathrm{b}}\left\langle I_{r}(t, \tau)\right\rangle_{\mathrm{b}}\left\langle\kappa_{j}\right\rangle_{\mathrm{b}}\left\langle I_{j}(t, \tau)\right\rangle_{\mathrm{b}}\right\} \\
& =\frac{\delta_{t s}}{\tau^{2}} \sum_{r=1}^{N}\left\{\left\langle\kappa_{r}^{2}\right\rangle_{\mathrm{b}}\left\langle I_{r}(t, \tau)\right\rangle_{\mathrm{b}}-\frac{\tau^{2}}{N} \mu^{2}(P)+\right. \\
& \left.\left\langle I_{r}(t, \tau)\right\rangle_{\mathrm{b}}\left\langle\kappa_{r}\right\rangle_{\mathrm{b}} \sum_{j \neq r}^{N}\left\langle\kappa_{j}\right\rangle_{\mathrm{b}}\left\langle I_{j}(t, \tau)\right\rangle_{\mathrm{b}}\right\} \\
& \stackrel{27}{=} \frac{\delta_{t s}}{\tau^{2}} \sum_{r=1}^{N}\left\{\left\langle\kappa_{r}^{2}\right\rangle_{\mathrm{b}}\left\langle I_{r}(t, \tau)\right\rangle_{\mathrm{b}}-\frac{\tau^{2}}{N} \mu^{2}(P)+\right. \\
& \left.\frac{\tau}{N} \mu(P) \sum_{j \neq r}^{N} \frac{\tau}{N} \mu(P)\right\} .
\end{aligned}
$$


From this, we obtain

$$
\begin{aligned}
\sigma_{t s} & \simeq \frac{\delta_{t s}}{\tau} \frac{N}{\tau}\left\langle\kappa_{r}^{2}\right\rangle_{\mathrm{b}}\left\langle I_{r}(t, \tau)\right\rangle_{\mathrm{b}}-\frac{\delta_{t s}}{N} \mu^{2}(P) \\
& \stackrel{\text { 7a }}{=} \frac{\delta_{t s}}{\tau} n_{\mathrm{b}} C(P)\left\langle\kappa_{r}^{2}\right\rangle_{\mathrm{b}}-\frac{\delta_{t s}}{N} \mu^{2}(P) .
\end{aligned}
$$

The last term vanishes, if we consider the thermodynamic limit (TDL) of an infinite heat bath, i.e., $N, L \rightarrow \infty$ such that $n_{\mathrm{b}}=N / L=$ constant. Thus, reinserting the explicit expression for $\kappa_{r}$, we obtain in this limit

$$
\sigma_{t s} \rightarrow \frac{\delta_{t s}}{\tau} n_{\mathrm{b}} C(P)\left\langle\left(\frac{2}{1-u_{r}^{2}} \frac{E}{E+\epsilon_{r}} p_{r}\right)^{2}\right\rangle_{\mathrm{b}}
$$

In principle, any higher correlation function can be calculated in the same manner. It is also evident that the noise force is non-Gaussian, because the relativistic bath distribution $f_{\mathrm{b}}\left(p_{r}\right)$ that determines the averages $\langle\cdot\rangle_{\mathrm{b}}-$ and, thus, the noise correlations - is non-Gaussian.

Finally, by substituting the averaged friction coefficient

$$
\nu(P)=n_{\mathrm{b}} C(P)\left\langle\frac{2}{1-u_{r}^{2}} \frac{\epsilon_{r}}{E+\epsilon_{r}}\right\rangle_{\mathrm{b}}
$$

for the square bracket term in Eq. (22), imposing the TDL for the bath and letting $\tau \rightarrow 0$ in Eq. (22), we obtain the relativistic LE

$$
\dot{P}=-\nu(P) P+\chi(t),
$$

where, in view of approximation (25), the non-Gaussian noise force $\chi$ is characterized by

$$
\langle\chi(t)\rangle_{\mathrm{b}}=n_{\mathrm{b}} C(P)\left\langle\frac{2}{1-u_{r}^{2}} \frac{E}{E+\epsilon_{r}} p_{r}\right\rangle_{\mathrm{b}},
$$

and

$$
\langle\chi(t) \chi(s)\rangle_{\mathrm{b}}-\langle\chi(t)\rangle_{\mathrm{b}}\langle\chi(s)\rangle_{\mathrm{b}}=2 D(P) \delta(t-s),
$$

with (momentum-space) diffusion coefficient

$$
D(P)=\frac{n_{\mathrm{b}}}{2} C(P)\left\langle\left(\frac{2}{1-u_{r}^{2}} \frac{E}{E+\epsilon_{r}} p_{r}\right)^{2}\right\rangle_{\mathrm{b}} .
$$

In Fig. 4 the ratio $D(P) /\left[n_{\mathrm{b}} C(P)\right]$ is plotted for the same parameters as in Figs. 1 and 3 As it is evident from the diagrams, this quantity increases with temperature $T$ and absolute momentum $P$ of the Brownian particle.

\section{RESUME}

We conclude the derivation of the relativistic LE with a set of general remarks:

(i) While deriving the relativistic LE (32), we made use of the stationarity, independence, and homogeneity of the bath distribution (17a); we did not, however, rely
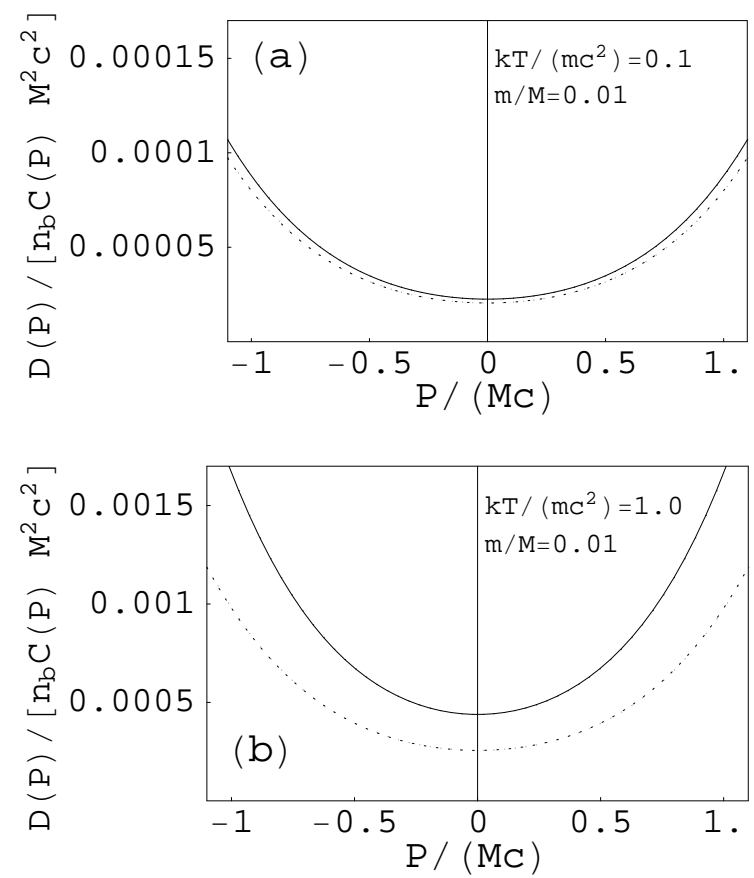

FIG. 4: Relativistic diffusion coefficients $D(P)$ calculated numerically for different heat bath distributions $f_{\mathrm{b}}^{1}(p)$ for (a) weakly and (b) strongly relativistic heat. The solid lines refer to a standard Jüttner distribution with $\eta=0$, and the dotted lines to $\eta=1$ in Eq. (17b).

on the specific properties of the marginal momentum PDF. Hence, the above results hold true for arbitrary one-particle momentum distributions $f_{\mathrm{b}}^{1}(p)$.

(ii) In order to be able to use the LEs derived above, one still needs to calculate the mean collision rate $C(P) / L$, which is determined by Eq. (7b); cf. in the Appendix. We also emphasize once again that the approximation (25), leading to the appearance of $C(P)$, can in principle be omitted (in the non-relativistic as well as in the relativistic case). More precise results for friction coefficients and noise correlations can then be extracted from Eq. An in the Appendix.

(iii) The stochastic force $\chi(t)$ in the Eq. (32) is $\delta$ correlated (memory-free), but non-Gaussian; i.e., in order to completely specify the stochastic process one actually has to determine all higher order correlation functions. This is practically unfeasible. Therefore, in numerical studies and/or practical applications, one could attempt to use the Gaussian approximation $\chi(t) \simeq \sqrt{2 D(P)} \zeta(t)$ instead, where $\zeta$ corresponds to standardized white noise with $\langle\zeta(t)\rangle=0$ and $\langle\zeta(t) \zeta(s)\rangle=$ $\delta(t-s)$. However, in general, it cannot be expected that such a 'truncated' LE yields the correct relaxation behavior and/or the correct stationary solution [59, 62]. Furthermore, also under such simplifying approximations, the results will depend on the choice of the discretization rule $50,53,54,55,56,57,58,59,60]$ because of the multiplicative coupling between $\sqrt{2 D(P)}$ and $\zeta(t)$. Loosely 
speaking, this discretization dilemma is the price that one has to pay for mapping the large number of collisions between $t$ and $t+\tau$ into a single instant of time. Our experience with the non-relativistic LE (cf. remarks at the end of Sec. IIB suggests that the "transport-form", i.e. the post-point discretization rule [50, 58, 59, 60] should be preferable in the relativistic case as well.

(iv) In principle, it should be straightforward to generalize the above approach to higher space dimensions, by expressing the momentum vector after the collision, $\hat{\boldsymbol{P}}$, in terms of the momenta before the collision, $\boldsymbol{P}$ and $\boldsymbol{p}$, analogous to Eq. (21). In the $2 d$ or $3 d$-case, complications may arise mostly due to the fact that one also has take into account the corresponding collision angles and cross-sections (e.g., when determining the collision rates; cf. comments at the end of the Appendix).

(v) According to our above results, the previously proposed 'relativistic' LEs [21, 26, 28, 29] should be viewed as approximations, which can be useful for generating/simulating ensembles of relativistic particles in a simple manner. It is also evident now why these earlier approaches have intrinsic limits. Debbasch et al. 21] have postulated that also the relativistic force is ordinary Gaussian white noise with a constant amplitude $D$, whereas we in our prior works [28, 29] assumed the validity of the momentum-independent Stokes approximation in the co-moving rest-frame of the Brownian particle. As follows from the derivation presented here, neither of these assumptions is appropriate if one properly takes into account both the relativistic conservation laws and the relativistic momentum distributions of the heat bath particles. Thus, if one is interested in the actual relaxation dynamics of relativistic Brownian particles in a relativistic environment (heat bath), then the relativistic LE (32) is expected to provide the physically more correct stochastic description.

\section{Acknowledgments}

J. D. would like to thank S. Hilbert for numerous helpful discussions.
[1] A. Einstein, Ann. Phys. (Leipzig) 17, 549 (1905).

[2] A. Einstein and M. von Smoluchowski, Untersuchungen über die Theorie der Brownschen Bewegung/Abhandlungen über die Brownsche Bewegung und verwandte Erscheinungen, vol. 199 (Harri Deutsch, Frankfurt, 1999), 3rd ed

[3] G. E. Uhlenbeck and L. S. Ornstein, Phys. Rev. 36, 823 (1930).

[4] S. Chandrasekhar, Rev. Mod. Phys. 15, 1 (1943).

[5] M. C. Wang and G. E. Uhlenbeck, Rev. Mod. Phys. 17, 323 (1945).

[6] P. Hänggi and P. Jung, Adv. Chem. Phys. 89, 239 (1995).

[7] P. Hänggi and F. Marchesoni, Chaos 15, 026101 (2005).

[8] E. Frey and K. Kroy, Ann. Phys. (Leipzig) 14, 20 (2005).

[9] P. Hänggi, F. Marchesoni, and F. Nori, Ann. Phys. (Leipzig) 14, 51 (2005).

[10] C. Lämmerzahl, Ann. Phys. (Leipzig) 14, 71 (2005).

[11] P. Reimann and P. Hänggi, Appl. Phys. A 75, 169 (2002).

[12] R. Bartussek, P. Reimann, and P. Hänggi, Phys. Rev. Lett. 76, 1166 (1996).

[13] G. Schay, Ph.D. thesis, Princeton University (1961), available through University Microfilms, Ann Arbor, Michigan, https://wwwlib.umi.com.

[14] R. Hakim, J. Math. Phys. 6, 1482 (1965).

[15] R. M. Dudley, Arkiv för Matematik 6, 241 (1965).

[16] F. Guerra and P. Ruggiero, Lett. Nouvo Cimento 23, 529 (1978).

[17] T. H. Boyer, Phys. Rev. D 19, 1112 (1979).

[18] U. Ben-Ya'acov, Phys. Rev. D 23, 1441 (1981).

[19] L. M. Morato and L. Viola, J. Math. Phys. 36, 4691 (1995), erratum, ibid. 37, 4769 (1996).

[20] A. Posilicano, Lett. in Math. Phys. 42, 85 (1997).

[21] F. Debbasch, K. Mallick, and J. P. Rivet, J. Stat. Phys 88, 945 (1997).

[22] P. Kostädt and M. Liu, Phys. Rev. D 62, 023003 (2000).
[23] G. Wolschin, Phys. Rev. C 69, 024906 (2004).

[24] F. Debbasch, J. Math. Phys. 45, 2744 (2004).

[25] T. Koide, Phys. Rev. E 72, 026135 (2005).

[26] R. Zygadlo, Phys. Lett. A 345, 323 (2005).

[27] D. Rapoport, Found. of Physics 35, 1205 (2005).

[28] J. Dunkel and P. Hänggi, Phys. Rev. E 71, 016124 (2005).

[29] J. Dunkel and P. Hänggi, Phys. Rev. E 72, 036106 (2005).

[30] A. Fingerle (2006), arXiv:cond-mat/0601303.

[31] J. Dunkel and P. Hänggi, Submitted to Physica A (2006), arXiv:cond-mat/0606487.

[32] M. Abdel-Aziz and S. Gavin, Phys. Rev. C 70, 034905 (2004).

[33] M. Abdel-Aziz and S. Gavin, J. Phys. G: Nucl. Phys. 31, 77 (2005).

[34] H. van Hees, V. Greco, and R. Rapp, Phys. Rev. C 73, 034913 (2006).

[35] H. van Hees, V. Greco, and R. Rapp (2006), arXiv:hep$\mathrm{ph} / 0601166$.

[36] M. E. Dieckmann, L. Drury, and P. K. Shukla, New J. Phys. 8, 40 (2006).

[37] M. Marti, R. A. Fonseca, and L. O. Silva (2006), "A collision module for OSIRIS", P5.015, 32nd European Physical Society Conference on Plasma Physics, Rome.

[38] A. Duviryuk, A. Nazarenko, and V. Tretyak, Cond. Mat. Phys. 4, 5 (2001).

[39] E. Lehmann, J. Math. Phys. 47, 023303 (2006).

[40] P. Pechukas, in Large-Scale Molecular Systems, edited by W. Gans, A. Blumen, and A. Amann (Plenum, New York, 1991), NATO ASI B258, p. 123.

[41] S. Tsonchev and P. Pechukas, Phys. Rev. E 61, 6171 (2000).

[42] N. Wiener, J. Math. Phys. 58, 131 (1923).

[43] N. N. Bogolyubov, Publ. Acad. Sci. Ukr. SSR Kiev pp. 115-137 (1945), in Russian.

[44] V. B. Magalinskii, Sov. Phys. JETP 9, 1381 (1959). 
[45] G. W. Ford, M. Kac, and P. Mazur, J. Math. Phys. 6, 505 (1965).

[46] R. Zwanzig, J. Stat. Phys. 9, 215 (1973).

[47] P. Hänggi, in Stochastic dynamics (Springer, Heidelberg, 1997), pp. 15-22.

[48] P. Hänggi and G. L. Ingold, Chaos 15, 026105 (2005).

[49] P. Hänggi, P. Talkner, and M. Borkovec, Rev. Mod. Phys. 62, 251 (1990).

[50] P. Hänggi and H. Thomas, Phys. Rep. 88, 207 (1982).

[51] N. G. Van Kampen, Stochastic Processes in Physics and Chemistry (North-Holland Personal Library, Amsterdam, 2003).

[52] R. Becker, Theory of heat (Springer, New York, 1967).

[53] K. Ito, Proc. Imp. Acad. Tokyo 20, 519 (1944).

[54] K. Ito, Mem. Amer. Mathem. Soc. 4, 51 (1951).

[55] D. Fisk, Ph.D thesis, Michigan State University, Dept. of Statistics (1963).

[56] R. L. Stratonovich, Vestnik Moskov. Univ., Ser. I: Mat., Mekh. 1, 3 (1964).

[57] R. L. Stratonovich, SIAM J. Control 4, 362 (1966).

[58] P. Hänggi, Helv. Phys. Acta 51, 183 (1978).

[59] P. Hänggi, Helv. Phys. Acta 53, 491 (1980).

[60] Y. L. Klimontovich, Physics-Uspekhi 37, 737 (1994).

[61] F. Jüttner, Ann. Phys. (Leipzig) 34, 856 (1911).

[62] P. Hänggi, Phys. Rev. A 25, 1130 (1982).

[63] For the non-relativistic Brownian motion, this problem was solved by Bogolyubov [43], Magalinskii [44], Ford et al. 45] and Zwanzig [46], who considered a bath of harmonic oscillators.

\section{APPENDIX A: CALCULATION OF THE COLLISION RATE}

We aim to derive an explicit expression for the expectation value $\left\langle I_{r}(t, \tau)\right\rangle_{\mathrm{b}}$ in the limit $\tau \rightarrow 0$, as, e.g., required in Eqs. (10).

By definition, the function $I_{r}(t, \tau) \in\{0,1\}$ indicates whether or not the Brownian particle has collided with the heat bath particle $r$ during the time interval $[t, t+\tau]$. The positions of the Brownian and heat bath particle at time $t$ are denoted by $X$ and $x_{r}$, respectively. Ignoring the possibility of a collision, for small enough $\tau$, the new positions at time $t+\tau$ would be given by

$$
X^{\prime}=X+V \tau, \quad x_{r}^{\prime}=x_{r}+v_{r} \tau,
$$

where $V$ and $v_{r}$ are the velocities. Then, the indicator function $I_{r}(t, \tau)$ can be explicitly represented as

$$
\begin{aligned}
I_{r}(t, \tau)= & \Theta\left(X-x_{r}\right) \Theta\left(x_{r}^{\prime}-X^{\prime}\right) \Theta\left(v_{r}-V\right)+ \\
& \Theta\left(x_{r}-X\right) \Theta\left(X^{\prime}-x_{r}^{\prime}\right) \Theta\left(V-v_{r}\right),
\end{aligned}
$$

where $\Theta(x)$ is the Heaviside-function, defined by

$$
\Theta(x)= \begin{cases}0, & x<0 \\ 1 / 2, & x=0 \\ 1, & x>0\end{cases}
$$

The first (second) summand in Eq. A2 refers to the initial configuration, where the heat bath particle is located at the left (right) side of the Brownian particle. Let us list some properties of the collision indicator $I_{r}(t, \tau)$.

First we note that $I_{r}(t, \tau)$ is idempotent, i.e.,

$$
I_{r}^{j}(t, \tau)=I_{r}(t, \tau)
$$

holds for $j=1,2 \ldots$. Furthermore, for $\tau \rightarrow 0$, we have

$$
I_{r}(t, 0)=0 .
$$

Accordingly, the Taylor-expansion at $\tau=0$ gives

$$
I_{r}(t, \tau) \simeq\left[\frac{\partial I_{r}}{\partial \tau}(t, 0)\right] \tau
$$

In order to determine $\left\langle\frac{\partial I_{r}}{\partial \tau}(t, 0)\right\rangle_{\mathrm{b}}$, we note that

$$
\begin{aligned}
\left.\frac{\partial}{\partial \tau} \Theta\left(x_{r}^{\prime}-X^{\prime}\right)\right|_{\tau=0} & =\left.\frac{\partial}{\partial \tau} \Theta\left(x_{r}-X+\left(v_{r}-V\right) \tau\right)\right|_{\tau=0} \\
& =\left.\left(v_{r}-V\right) \delta\left(x_{r}-X+\left(v_{r}-V\right) \tau\right)\right|_{\tau=0} \\
& =\left(v_{r}-V\right) \delta\left(x_{r}-X\right)
\end{aligned}
$$

and, analogously,

$$
\begin{aligned}
\left.\frac{\partial}{\partial \tau} \Theta\left(X^{\prime}-x_{r}^{\prime}\right)\right|_{\tau=0} & =\left.\frac{\partial}{\partial \tau} \Theta\left(X-x_{r}+\left(V-v_{r}\right) \tau\right)\right|_{\tau=0} \\
& =\left.\left(V-v_{r}\right) \delta\left(X-x_{r}+\left(V-v_{r}\right) \tau\right)\right|_{\tau=0} \\
& =\left(V-v_{r}\right) \delta\left(X-x_{r}\right) .
\end{aligned}
$$

Hence, we find

$$
\begin{aligned}
\frac{\partial I_{r}}{\partial \tau}(t, 0)= & \left(v_{r}-V\right) \Theta\left(X-x_{r}\right) \delta\left(x_{r}-X\right) \Theta\left(v_{r}-V\right)+ \\
& \left(V-v_{r}\right) \Theta\left(x_{r}-X\right) \delta\left(X-x_{r}\right) \Theta\left(V-v_{r}\right), \\
= & \Theta(0)\left(v_{r}-V\right) \delta\left(x_{r}-X\right) \Theta\left(v_{r}-V\right)+ \\
& \Theta(0)\left(V-v_{r}\right) \delta\left(X-x_{r}\right) \Theta\left(V-v_{r}\right),
\end{aligned}
$$

and, with $\Theta(0)=1 / 2$, the useful result

$$
\begin{aligned}
\frac{\partial I_{r}}{\partial \tau}(t, 0)= & \frac{1}{2}\left(v_{r}-V\right) \delta\left(x_{r}-X\right) \Theta\left(v_{r}-V\right)+ \\
& \frac{1}{2}\left(V-v_{r}\right) \delta\left(X-x_{r}\right) \Theta\left(V-v_{r}\right) .
\end{aligned}
$$

Now let us consider a spatially homogeneous oneparticle bath distribution of the form

$$
\tilde{f}_{\mathrm{b}}^{1}\left(x_{r}, v_{r}\right)=\frac{1}{L} \tilde{f}_{\mathrm{b}}^{1}\left(v_{r}\right) \begin{cases}1, & x_{r} \in[-L / 2, L / 2] \\ 0, & x_{r} \notin[-L / 2, L / 2]\end{cases}
$$

and some function $\tilde{G}\left(x_{r}, v_{r}\right)$ such that the expectation value $\left\langle\tilde{G}\left(x_{r}, v_{r}\right)\right\rangle_{\mathrm{b}}$ exists. We are interested in expectations of the form

$$
\left\langle\tilde{G}\left(x_{r}, v_{r}\right) I_{r}^{j}(t, \tau)\right\rangle_{\mathrm{b}} \stackrel{\text { A4a }}{=}\left\langle\tilde{G}\left(x_{r}, v_{r}\right) I_{r}(t, \tau)\right\rangle_{\mathrm{b}},
$$


as required for calculating the mean value of the stochastic force and its higher correlation functions [e.g., compare first line of Eq. (29)]. For small $\tau$, we may truncate the Taylor expansion after the linear term, yielding

$$
\left\langle\tilde{G}\left(x_{r}, v_{r}\right) I_{r}(t, \tau)\right\rangle_{\mathrm{b}} \simeq\left\langle\tilde{G}\left(x_{r}, v_{r}\right) \frac{\partial I_{r}}{\partial \tau}(t, 0)\right\rangle_{\mathrm{b}} \tau .
$$

Making use of the result $\mathrm{A} 4 \mathrm{~d}$, the mean value on the rhs. is given by

$$
\begin{array}{r}
\left\langle\tilde{G}\left(x_{r}, v_{r}\right) \frac{\partial I_{r}}{\partial \tau}(t, 0)\right\rangle_{\mathrm{b}}= \\
\frac{1}{2 L} \int_{V}^{\infty} \mathrm{d} v_{r}\left(v_{r}-V\right) \times \\
\tilde{G}\left(X, v_{r}\right) \tilde{f}_{\mathrm{b}}^{1}\left(v_{r}\right)+ \\
\frac{1}{2 L} \int_{-\infty}^{V} \mathrm{~d} v_{r}\left(V-v_{r}\right) \times \\
\tilde{G}\left(X, v_{r}\right) \tilde{f}_{\mathrm{b}}^{1}\left(v_{r}\right) .
\end{array}
$$

In particular, by choosing $\tilde{G}\left(x_{r}, v_{r}\right) \equiv 1$, we find the collision rate

$$
\lim _{\tau \rightarrow 0} \frac{\left\langle I_{r}(t, \tau)\right\rangle_{\mathrm{b}}}{\tau}=\left\langle\frac{\partial I_{r}}{\partial \tau}(t, 0)\right\rangle_{\mathrm{b}}=\frac{1}{L} \tilde{C}(V),
$$

where

$$
\begin{aligned}
\tilde{C}(V) \equiv & \frac{1}{2} \int_{V}^{\infty} \mathrm{d} v_{r}\left(v_{r}-V\right) \tilde{f}_{\mathrm{b}}^{1}\left(v_{r}\right)+ \\
& \frac{1}{2} \int_{-\infty}^{V} \mathrm{~d} v_{r}\left(V-v_{r}\right) \tilde{f}_{\mathrm{b}}^{1}\left(v_{r}\right) .
\end{aligned}
$$

The following comments are in order:

(i) The above derivation is valid for both nonrelativistic and relativistic heat bath distributions $\tilde{f}_{\mathrm{b}}^{1}\left(v_{r}\right)$. Upon identifying $C(P) \equiv \tilde{C}(V(P))$, where $P$ is the momentum of the Brownian particle, we obtain the rigorous justification for Eq. (7a). However, in the non-relativistic case we have $V=P / M$, whereas in the relativistic case $V=P /\left(M^{2}+P^{2}\right)^{1 / 2}$. Additionally, we note that the support interval of the relativistic velocity distribution $\tilde{f}_{\mathrm{b}}^{1}\left(v_{r}\right)$ is given by $[-c, c]$, which determines the effective upper and lower integral boundaries in Eq. A7b.

(ii) Given a certain bath distribution $\tilde{f}_{\mathrm{b}}^{1}\left(v_{r}\right)$, the exact result (A6) allows for evaluating the quality of the product approximations (8) and (25), respectively.

(iii) The Stokes approximation corresponds to setting $V=0$ in Eq. A7b, yielding

$$
\tilde{C}(0)=\frac{1}{2} \int_{-\infty}^{\infty} \mathrm{d} v_{r}\left|v_{r}\right| \tilde{f}_{\mathrm{b}}^{1}\left(v_{r}\right)
$$

This shows that the Stokes approximation is useful for slow Brownian particles, but inappropriate at high velocities.

(iv) It is in principle possible to apply the same procedure to higher space dimensions, but then the expression (A2) for the indicator unction has to be modified accordingly (e.g., by taking into account the geometric shape of the Brownian particle). As a consequence, analytic calculations will become much more difficult. 